\title{
A Modified Plasma Arc Gas Condensation Technique to Synthesize Nanocrystalline Tungsten Oxide Powders
}

\author{
Cherng-Yuh Su${ }^{1}$, Chung-Kwei Lin $^{2}$ and Chia-Wei Cheng ${ }^{3}$ \\ ${ }^{1}$ Department of Mechanical Engineering, National Taipei University of Technology, Taipei, Taiwan \\ ${ }^{2}$ Department of Materials Science and Engineering, Feng-Chia University, Taichung, Taiwan \\ ${ }^{3}$ Department of Mechanical Engineering, Da-Yeh University, Chang-Hwa, Taiwan
}

\begin{abstract}
In the present study, nanocrystalline tungsten oxide powders were prepared by a modified plasma arc gas condensation technique where a gas nozzle was introduced to provide blowing gas. With the aid of the blowing gases, nanocrystalline tungsten oxide powders can be prepared under various chamber pressures ranged from 4.9 to $101.3 \mathrm{kPa}$. The mean grain size and powder production rate of the as-prepared nanocrystalline $\mathrm{WO}_{3}$ powders increased with increasing chamber pressure. For an increasing chamber pressure from 4.9 to $101.3 \mathrm{kPa}$, asignificant increase in powder production rate from 0.374 to $13.658 \mathrm{~g} / \mathrm{h}$ can be noticed, while the mean grain size only enlarged acceptably from 5.9 to $15.7 \mathrm{~nm}$. Meanwhile, by controlling the partial oxygen pressure of the mixed gas, nanocrystalline blue tungsten oxide powders can be prepared successfully. The blowing mixed gas from the nozzle not only suppressed the nucleation and growth for powders from the gas phase, but can be used to prepare stoichiometric or nonstoichiometric tungsten oxide powders.
\end{abstract}

(Received December 10, 2004; Accepted March 8, 2005; Published May 15, 2005)

Keywords: plasma arc gas condensation technique, nanocrystalline $\mathrm{WO}_{3}$ powders, nonstoichiometric tungsten oxide

\section{Introduction}

Inert gas condensation technique ${ }^{1)}$ has been widely used to prepare nanocrystalline powders including metals, alloys, and ceramics. In a typical gas condensation technique, the raw material is evaporated by various heat sources such as electrical resistance under a pressure usually $\sim 1.33 \times$ $10^{-3} \mathrm{~Pa}$ or better. If a high temperature arc or plasma is used as the heating source, the chamber pressure can be in the range of 13.3 to $53.3 \mathrm{kPa}^{2,3)}$ that is higher than that of electrical resistance. The limited evaporation of target and fast consumption of electrode, however, restrict the use of arc heat source in higher pressures. For the current modified plasma arc gas condensation technique with the aid of blowing gas ${ }^{2)}$ the feasibility to synthesize nanocrystalline powders at a chamber pressure reaching $101.3 \mathrm{kPa}$ can be achieved.

Various metals, alloys and ceramics can be prepared by the modified plasma arc gas condensation technique. Among these materials, nanocrystalline oxides powders are of great interests due to their unique optical ${ }^{4)}$ and electrical properties,${ }^{5)}$ etc. Thus, tungsten oxide is chosen as a prototype and presented in the current study. Nanocrystalline tungsten oxide powders exhibit unique optical, ${ }^{4)}$ electrical, ${ }^{5)}$ electrochromic, ${ }^{6-8)}$ catalytic, $^{9)}$ and gas-sensing properties. ${ }^{10,11)}$ Meanwhile, it can also be used to prepare polymer-matrixcomposites. In the present study, we plan to use the modified plasma arc gas condensation technique to synthesize nanocrystalline tungsten oxide powders in a wide range of chamber pressure with the aid of blowing gas. In addition, we will alter the oxygen partial pressure in the mixed gas to create an oxygen deficient environment. Thus we can prepare nonstoichiometric nanocrystalline tungsten oxide powders. ${ }^{12)}$

\section{Experimental Procedures}

Figure 1 shows the schematic illustration of the modified

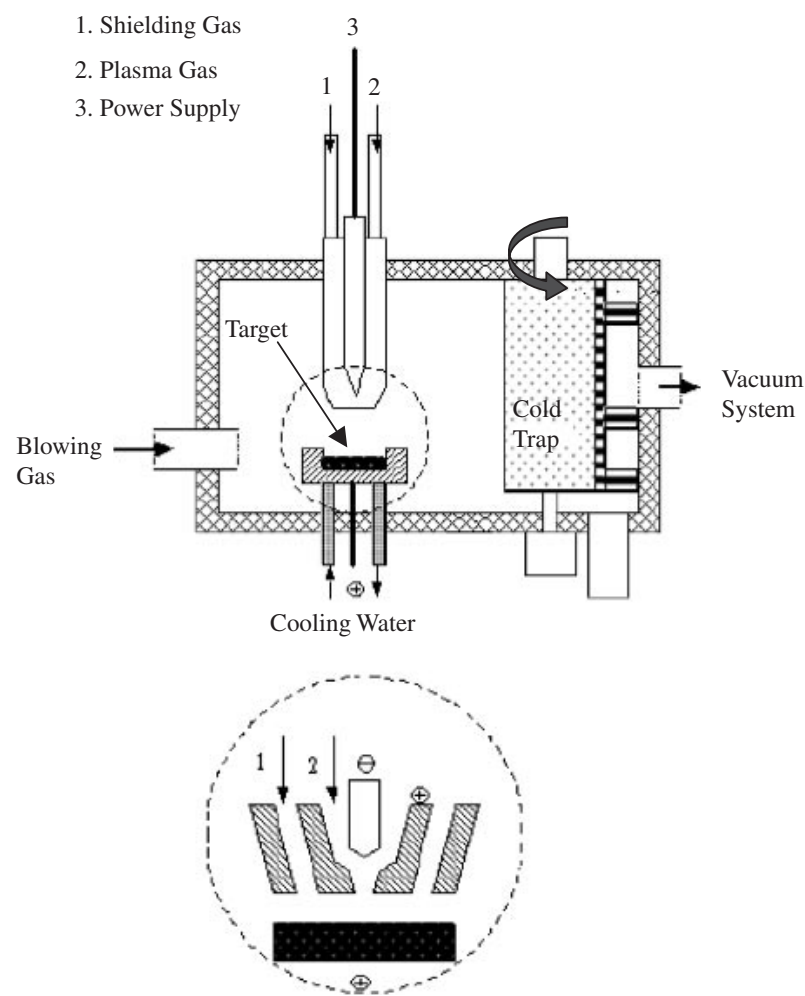

Fig. 1 Schematic of plasma arc gas condensation equipment.

plasma arc gas condensation system used in the present study. The tungsten raw material (purity $=99.95 \%$ ) was heated by a water-cooled plasma gun (model: PWM-300, Thermadyne International, USA), evaporated in the controlled atmosphere, and nanocrystalline $\mathrm{WO}_{3}$ powders can be collected on the cold trap. There are several parameters such as plasma power input (current and voltage), and chamber atmosphere (gas type and pressure) can be varied to prepare nanocrystalline powders with different mean particle size and 
Table 1 Processing parameters for plasma arc gas condensation technique.

\begin{tabular}{cc}
\hline Processing Parameters & Value \\
\hline Plasma Current & $90 \mathrm{~A}$ \\
\hline Plasma Voltage & $26 \mathrm{~V}$ \\
\hline Setback & $0.093 \mathrm{in}$ \\
\hline Shielding Gas (Ar) & $6 \sim 8 \mathrm{SCFH}$ \\
\hline Plasma Gas (Ar) & $1.4 \sim 1.6 \mathrm{SCFH}$ \\
\hline Arc Length & $1: 1$ \\
\hline Gas Ratio (Ar: $\left.\mathrm{O}_{2}\right)$ & $99.99 \%$ \\
\hline Gas Purity (Ar or $\left.\mathrm{O}_{2}\right)$ &
\end{tabular}

distribution. In contrast to a typical gas condensation equipment where the atmosphere is usually pumped to a high vacuum (say $1.33 \times 10^{-3} \mathrm{~Pa}$ ) and backfilled with limited inert gas such as helium, the present innovated design can be used to prepare nanocrystaline powders at a much higher pressure. An inlet gas nozzle placed close to the plasma was used to introduce a gas flow of mixed argon and oxygen. Dynamic gas pressure equilibrium was carefully maintained at a designed constant value during the processing. Nanocrystalline $\mathrm{WO}_{3}$ powders were prepared under various chamber pressures ranged from 4.9 to $101.3 \mathrm{kPa}$. The processing parameters were summarized at Table 1 .

The as-received powders were then characterized by transmission electron microscopy (TEM), scanning electron microscopy (SEM), secondary particle size analysis, and Xray diffraction. The transmission electron microscopy images were examined by a HITACHI 800 TEM microscope. The particle size, shape and distribution can be obtained from the bright field image of TEM photographs. The morphology of the as-prepared powders was examined with a high-magnification field emission scanning electron microscope (ULVAC VPS-020). A Horiba LB-500 particle size analyzer was used to evaluate the agglomerated particle size distribution. Meanwhile, the phase identification of nanocrystalline powders was performed using a MAC MXP 18 X-ray diffractometer with a monochromatic $\mathrm{Cu} \mathrm{K} \alpha$ radiation.

\section{Results and Discussion}

\subsection{The effect of chamber pressure}

In the conventional inert gas condensation technique with electrical resistance as the heat source, ${ }^{13)}$ the sample chamber is usually pumped to a high (or even ultra-high) vacuum pressure better than $1.33 \times 10^{-3} \mathrm{~Pa}$, then backfilled with limited inert gas (such as helium or argon). If a plasma arc is used as the heat source, the chamber pressure is usually ranged from 13.3 to $53.3 \mathrm{kPa}^{2,3)}$ In the current study, the modified plasma arc processing can be operated under a much higher pressure (even $101.3 \mathrm{kPa}$ ) with a gas nozzle to provide the blowing gas. The excess plasma generated heat can be effectively removed by the blowing gas, thus the grain growth can be suppressed and nanocrystalline powders can be prepared successfully.

TEM observation is commonly used to examine the primary particle morphology of the nanocrystalline powders and Figure 2 shows the TEM images of the as-prepared
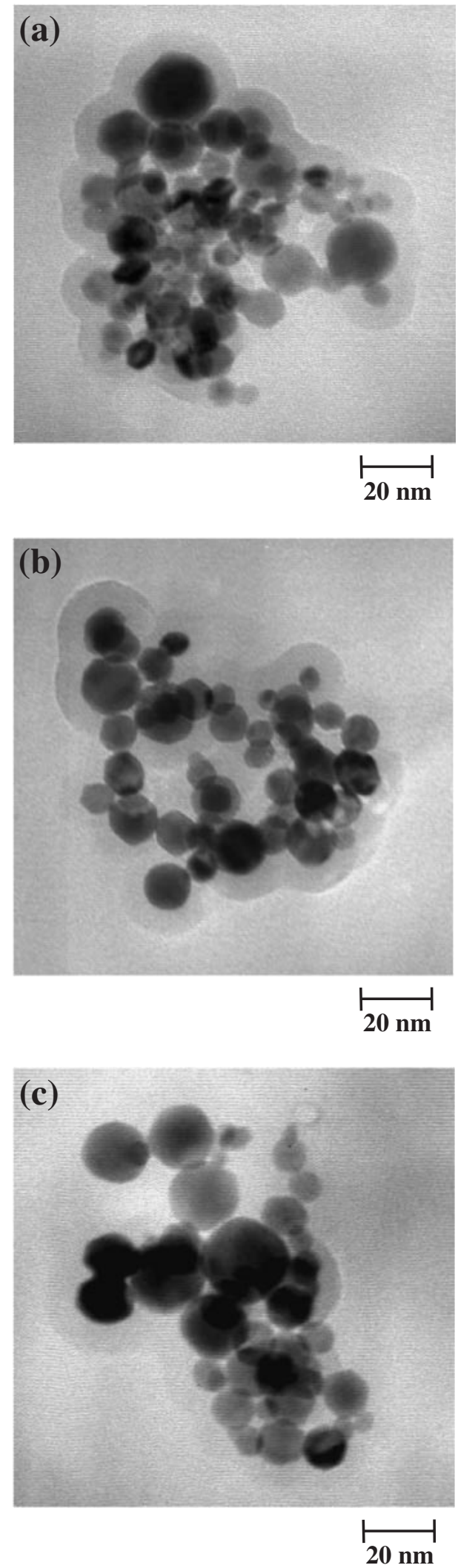

Fig. 2 TEM images of $\mathrm{NC} \mathrm{WO}_{3}$ powders prepared under a pressure of (a) 4.9 , (b) 20 , and (c) $40 \mathrm{kPa}$ respectively.

nanocrystalline tungsten oxide powders. It can be observed that the nanocrystalline $\mathrm{WO}_{3}$ powders prepared by the current modified plasma arc technique exhibited a mean grain size ranged from 6 to $18 \mathrm{~nm}$ for a pressure of 4.9 to $101.3 \mathrm{kPa}$ 
respectively. As shown in Fig. 2(a) $(4.9 \mathrm{kPa})$, nanocrystalline $\mathrm{WO}_{3}$ powders exhibited a mean grain size of $\sim 6 \mathrm{~nm}$. In addition to the major small spherical grains, a few large irregular shaped particles $(\sim 100 \mathrm{~nm}$, not shown in Fig. 2(a) due to magnification) can be observed. It is suspected that, at a relatively low pressure of $4.9 \mathrm{kPa}$, the limited blowing gas can not efficiently remove the plasma heat and thus an unavoidable grain growth occurred. ${ }^{2)}$ The blowing gas served as an agent to suppress the nucleation and growth of nanocrystalline powders initiated from the plasma heating of raw materials. When the chamber pressure increased to $20 \mathrm{kPa}$, nanocrystalline spherical $\mathrm{WO}_{3}$ powders $(\sim 10.7 \mathrm{~nm})$ with few large particles can be prepared, Fig. 2(b). The increasing chamber pressure provided by the blowing gas gradually reached an optimized condition between the heat generation (by plasma) and removal (by blowing gas). Using a relative high chamber pressure (higher than $40 \mathrm{kPa}$ ), nanocrystalline $\mathrm{WO}_{3}$ powders absent from large irregular shaped particles can be prepared at the expanse of slight grain growth.

Figure 3 shows the mean grain size of as-prepared nanocrystalline $\mathrm{WO}_{3}$ powders as a function of chamber pressure. It can be noted that the mean grain size increased with increasing chamber pressure. Under a low chamber pressure environment, a slight increase in chamber pressure from 4.9 to $10 \mathrm{kPa}$ enlarged clearly the grain size from $5.9 \mathrm{~nm}$ to $9.6 \mathrm{~nm}$ respectively (large irregular shaped grains were not counted in both cases). Thereafter the effect of chamber pressure on grain growth slowed down. This can be seen from the slope change as shown in Fig. 3. The grain size increased from $9.6 \mathrm{~nm}$ to $15.7 \mathrm{~nm}$ under a chamber pressure of $10 \mathrm{kPa}$ and $1010.3 \mathrm{kPa}$ respectively. The chamber pressure not only influenced the mean grain size of $\mathrm{WO}_{3}$ powders but also its production rate. Figure 4 shows the powder production rate as a function of chamber pressure where an almost linear relationship can be noted. It should be pointed out that there is a significant increase in powder production rate from 0.374 to $13.658 \mathrm{~g} / \mathrm{h}$ for a chamber pressure of $4.9 \mathrm{kPa}$ to $101.3 \mathrm{kPa}$ respectively. The production rate increased $\sim 37$ times at the expanse of $\sim$ three times increase in mean grain size. The

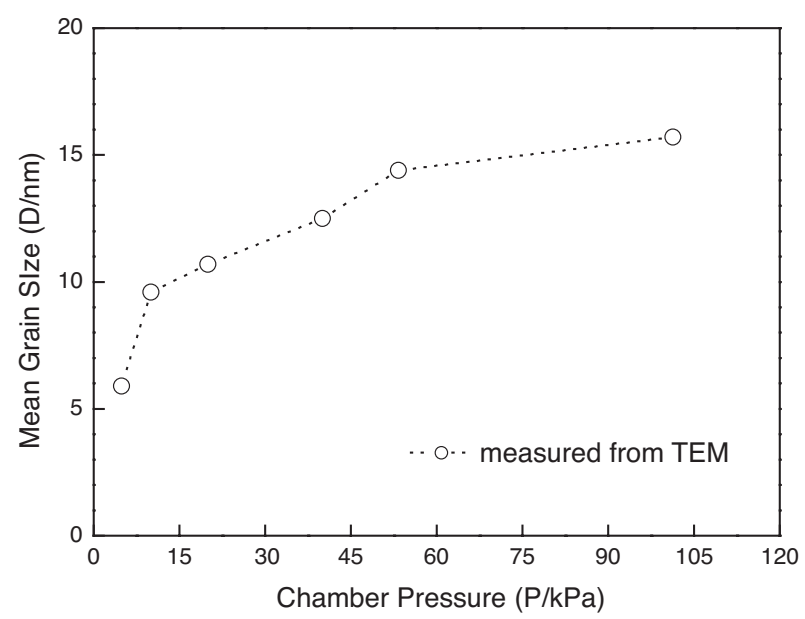

Fig. 3 The mean grain size of nanocrystalline $\mathrm{WO}_{3}$ powders as a function of chamber pressure.

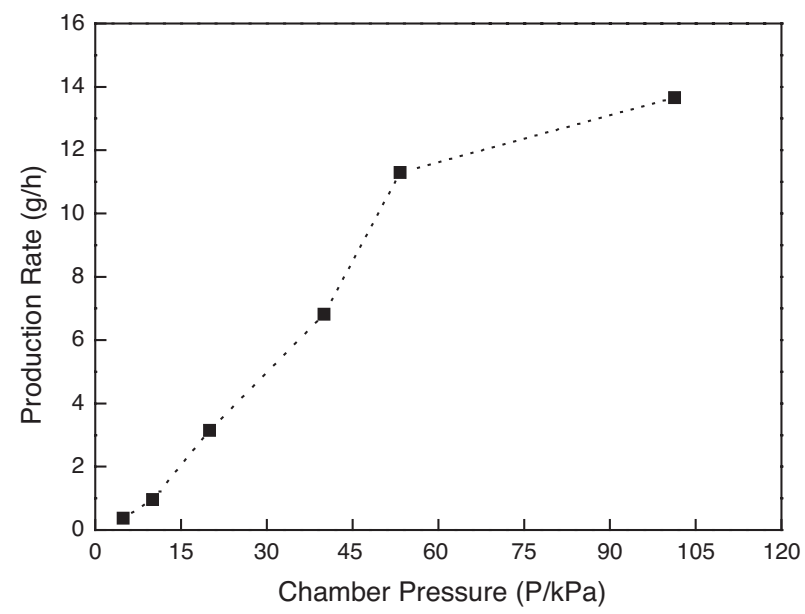

Fig. 4 The powder production rate as a function of chamber pressure.

Table 2 Powder grain size and production rate.

\begin{tabular}{cccc}
\hline $\begin{array}{c}\text { Chamber } \\
\text { Pressure } \\
(\mathrm{kPa})\end{array}$ & $\begin{array}{c}\text { Mean Grain } \\
\text { Size } \\
(\mathrm{nm})\end{array}$ & $\begin{array}{c}\text { Production } \\
\text { Rate } \\
(\mathrm{g} / \mathrm{h})\end{array}$ & $\begin{array}{c}\text { Standard } \\
\text { Deviation } \\
(\mathrm{nm})\end{array}$ \\
\hline 4.9 & 5.9 & 0.374 & - $^{*}$ \\
\hline 10 & 9.6 & 0.963 & - \\
\hline 20 & 10.7 & 3.147 & 8.01 \\
\hline 40 & 12.5 & 6.815 & 9.76 \\
\hline 53.3 & 14.4 & 11.291 & 10.8 \\
\hline 101.3 & 15.7 & 13.658 & 12.7 \\
\hline
\end{tabular}

*: The standard deviation is not calculated due to some large grains with a size of $\sim 100 \mathrm{~nm}$.

effect of blowing gas from the nozzle not only suppressed the nucleation and growth for powders from the gas phase, but did enhance the formation of nanocrystalline powders.

Table 2 summarizes the mean grain size and production rate of nanocrystalline $\mathrm{WO}_{3}$ powders prepared at various chamber pressures. Since low pressure usually favors the formation of nanocrystalline powders, it is suggested that nanocrystalline $\mathrm{WO}_{3}$ powders can be prepared at a pressure lower than $4.9 \mathrm{kPa}$. It should be noted that the plasma arc cannot be generated at low pressures. It is also possible, but not necessary, to prepare nanocrystalline powders at a pressure higher than $101.3 \mathrm{kPa}$. In the present study, we have demonstrated that nanocrystalline powders can be prepared successfully at a wide range of chamber pressure (4.9 to $101.3 \mathrm{kPa}$ ) with the modified plasma arc gas condensation technique.

Field emission scanning electron microscopy was used to examine the morphology of the as-prepared tungsten oxide powders. Large irregular shaped particles can be observed in Figs. 5(a) and 5(b) corresponding to a chamber pressure of 4.9 and $20 \mathrm{kPa}$ respectively. Meanwhile, it can be noted that nanocrystalline powders tended to agglomerate together after gas condensation. In addition to the primary grain size as observed by TEM (Fig. 2), the secondary particle size as observed by SEM (Fig. 5) due to agglomeration of primary particles is another important index for practical application 

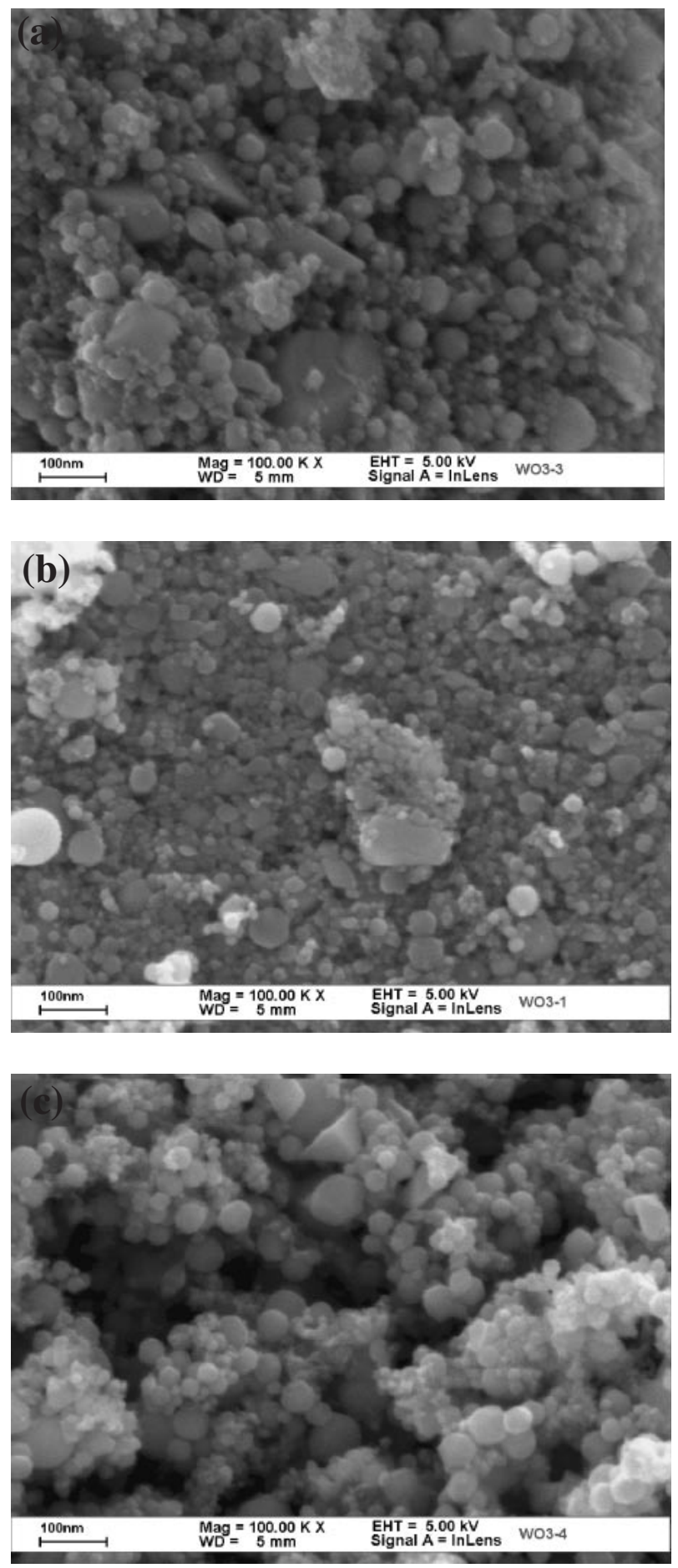

Fig. 5 SEM powder morphology observation of $\mathrm{NC} \mathrm{WO}_{3}$ powders prepared under a pressure of (a) 4.9 , (b) 20 , and (c) $40 \mathrm{kPa}$ respectively.

of the nanocrystalline powders. The secondary particle size distribution is examined by a laser-type particle size analyzer, where an appropriate amount of nanocrystalline powders were mixed into deionized water and followed by ultrasonic vibration. Some weak bonding among particles can be broken and the secondary particle size can be measured. As shown in Fig. 6, the gradually increasing particle size distribution can be noticed with increasing chamber pressure. This shows a similar trend, i.e., mean grain size increased with an increasing chamber pressure, as that of primary particle size.

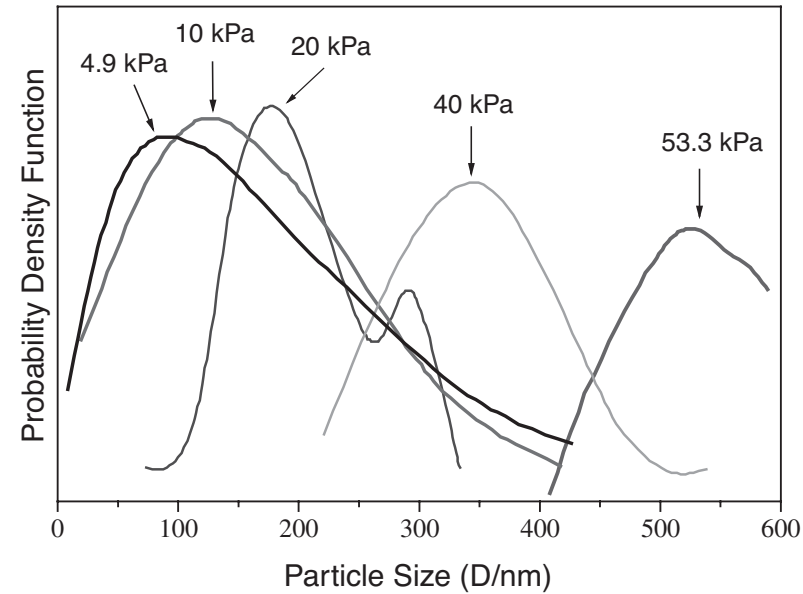

Fig. 6 Secondary particle size distribution of $\mathrm{NC}^{\mathrm{WO}_{3}}$ powders prepared under various chamber pressures.

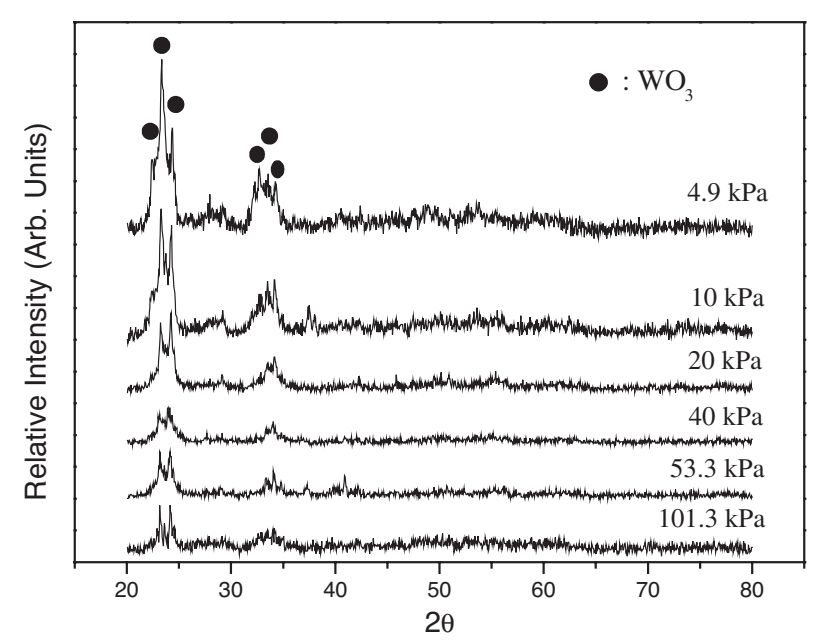

Fig. 7 X-ray diffraction patterns of $\mathrm{NC}^{-\mathrm{WO}_{3}}$ powders as a function of pressure.

\subsection{The effect of oxygen partial pressure}

Figure 7 shows the X-ray diffraction patterns of nanocrystalline tungsten oxide powders. $\mathrm{WO}_{3}$ phase exhibited for all as-prepared nanocrystalline $\mathrm{WO}_{3}$ powders. No significant change can be observed for these powders prepared under various chamber pressures. It should be pointed out that the more gas provided by the nozzle the more smoky powder dusts can be observed during the process. It is suggested that the oxygen in the mixed gas may play important role in the phenomenon. The smoky powder dusts were nanocrystalline $\mathrm{WO}_{3}$ powders that was confirmed by the TEM observations and X-ray diffraction patterns. While the amount of the smoky dusts can be revealed by the significant increase in powder production rate. Since the chamber pressure is kept in a dynamic equilibrium condition, the steady state amount of oxygen was supplied by the mixed gas and thus nanocrystalline $\mathrm{WO}_{3}$ powders can be prepared continuously. The major influence of chamber pressure is the production rate, not the grain size, as revealed by the present study.

Tungsten oxide is an electrochromic material. Nonstoichiometric tungsten oxide may exhibit unique optical and 


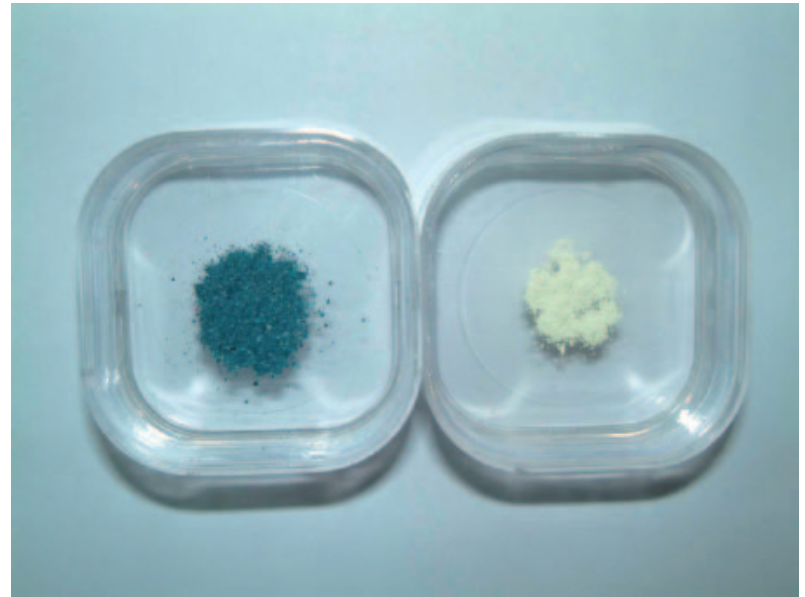

Fig. 8 Snapshot of blue and yellowish nanocrystalline tungsten oxide powders.

transport properties. Thus it will be interesting to investigate the nanocrystalline tungsten oxide powders prepared under an oxygen deficient environment. Simply by reducing the oxygen partial pressure in the mixed gas, blue nanocrystalline tungsten oxide powders can be obtained successfully. In contrast to the stoichiometric $\mathrm{WO}_{3}$ powders prepared under an $\mathrm{Ar} / \mathrm{O}_{2}$ gas ratio of $1: 1$, nonstoichiometric $\mathrm{WO}_{3}$ powders were obtained under an $\mathrm{Ar} / \mathrm{O}_{2}$ gas ratio of 100:1. Figure 8 shows a snapshot image of stoichiometric (right, yellowish color) and nonstoichiometric (left, blue color) nanocrystalline tungsten oxide powders. ${ }^{12)}$

In the present study, we have demonstrated that nanocrystalline tungsten oxide powders can be prepared by a modified plasma arc gas condensation technique with a pressure much higher than the conventional gas condensation method. Significant increase in the powder production rate can also be noticed. Meanwhile, by controlling the oxygen partial pressure, nanocrystalline nonstoichiometric blue tungsten oxide powders can be prepared. Not only the tungsten oxide powders but other materials including metals and ceramics can be synthesized. Practical industrial application of the modified plasma arc gas condensation technique is feasible.

\section{Conclusions}

A modified plasma arc gas condensation technique was used to prepare nanocrystalline tungsten oxide powders.
With the aid of the mixed blowing gases to maintain a dynamic equilibrium, nanocrystalline $\mathrm{WO}_{3}$ powders were prepared successfully under a chamber pressure in the range of 4.9 to $101.3 \mathrm{kPa}$. The mean grain size of the as-prepared powders ranged from 5.9 to $15.7 \mathrm{~nm}$. A significant increase in powder production rate from 0.374 to $13.658 \mathrm{~g} / \mathrm{h}$ can be noticed. Not only the primary particle size increased with increasing chamber pressure, but the secondary particle size showed a similar trend. X-ray diffraction results showed that all the as-prepared powders exhibited $\mathrm{WO}_{3}$ phase.

Nanocrystalline blue tungsten oxide powders, however, can be prepared successfully by controlling the partial oxygen pressure of the mixed gas. The blowing mixed gas from the nozzle not only suppressed the nucleation and growth for powders from the gas phase, but can be used to prepare stoichiometric or nonstoichiometric tungsten oxide powders.

\section{Acknowledgements}

The authors are grateful for the financial support of this work by the National Science Council of Taiwan, Republic of China under grant number NSC-93-2216-E-027-007.

\section{REFERENCES}

1) H. Gleiter: Progress in Mater. Sci. 33 (1989) 223-315.

2) M. H. Teng, J. J. Host, J. H. Hwang, B. R. Elliott, J. R. Weertman, T. O. Mason, V. P. David and D. L. Johnson: J. Mater. Res. 13 (1998) 25472555.

3) X. Devaux, F. Brochin, A. Dauscher, B. Lenoir, R. Martin-Lopez, H. Scherrer and S. Scherrer: Nanostructured Material 8 (1997) 137-147.

4) I. Turyan, U. O. Krasovec, B. Orel, T. Saraidorov, R. Reisfeld and D. Mandler: Adv. Mater. 12 (2000) 330-333.

5) E. K. H. Salje: Eu. J. Solid State Inorg. Chem. 31 (1994) 805-814.

6) C. G. Granqvist: Sol. Energy Mat. Sol. Cells 60 (2000) 201-262.

7) B. P. Jelle and G. Hagen: Sol. Energy Mat. Sol. Cells 58 (1999) 277286.

8) J. D. Guo, Y. J. J. Li and M. S. Whittingham: J. Power Sources 54 (1995) 461-464

9) F. B. Li, G. B. Gu, X. J. Li and H. F. Wan: J. Enviromental Sciences 13 (2001) 64-68.

10) W. M. Qu and W. Wlodarski: Sensors and Actuators B 64 (2000) 4248.

11) K. H. Lee, Y. K. Kang, W. J. Lee, J. J. Ho, K. H. Chen and K. S. Liao: Sensors and Actuators B 69 (2000) 96-99.

12) G. L. Frey, A. Rothschild, J. Sloan, R. Rosentsveig, R. Popovitz-Biro and R. Tenne: J. Solid State Chem. 162 (2001) 300-314.

13) B. Y. Wei, S. 1. Ho, F. Y. Chen and H. M. Lin: Surf. Coat. Tech. 166 (2003) 1-9. 